\title{
The Properties of a New Subclass of Harmonic Univalent Mappings
}

\author{
Zhi-Hong Liu and Ying-Chun Li \\ College of Mathematics, Honghe University, Mengzi, Yunnan 661199, China \\ Correspondence should be addressed to Zhi-Hong Liu; liuzhihongmath@163.com
}

Received 21 July 2013; Accepted 25 August 2013

Academic Editor: Irena Lasiecka

Copyright ( 2013 Z.-H. Liu and Y.-C. Li. This is an open access article distributed under the Creative Commons Attribution License, which permits unrestricted use, distribution, and reproduction in any medium, provided the original work is properly cited.

We introduced a new subclass of univalent harmonic functions defined by the shear construction in the present paper. First, we showed that the convolutions of two special subclass harmonic mappings are convex in the horizontal direction. Secondly, we proved a necessary and sufficient condition for the above subclass of harmonic mappings to be convex in the horizontal direction. We also presented some basic examples of univalent harmonic functions explaining the behavior of the image domains.

\section{Introduction}

Let $f=u+i v$ be a continuous complex-valued harmonic mapping in the open unit disc $\mathbb{D}=\{z \in \mathbb{C}:|z|<1\}$, where $u$ and $v$ are real-valued harmonic functions in $\mathbb{D}$. Such functions can be expressed as $f=h+\bar{g}$; here $h$ is known as the analytic part and $g$ the coanalytic part of $f$, respectively. The Jacobian of the mapping $f=h+\bar{g}$ is given by $\mathscr{J}_{f}=$ $\left|h^{\prime}\right|^{2}-\left|g^{\prime}\right|^{2}$. A necessary and sufficient condition (see [1] or [2]) for $f$ to be locally univalent and sense-preserving in $\mathbb{D}$ is that $\left|g^{\prime}\right|<\left|h^{\prime}\right|$, or equivalently, there exists an analytic complex dilatation $\omega(z)$ of $f$ such that

$$
|\omega(z)|=\left|\frac{g^{\prime}(z)}{h^{\prime}(z)}\right|<1 \quad\left(h^{\prime}(z) \neq 0, z \in \mathbb{D}\right) .
$$

We denote by $\mathcal{S}_{H}$ the class of harmonic, sense-preserving, and univalent mappings in $\mathbb{D}$, normalized by the conditions $f(0)=0$ and $f_{z}(0)=1$. Thus, a harmonic mapping in the class $\mathcal{S}_{H}$ can be expressed as $f=h+\bar{g}$, where

$$
h(z)=z+\sum_{n=2}^{\infty} a_{n} z^{n}, \quad g(z)=\sum_{n=1}^{\infty} b_{n} z^{n} .
$$

Let $\mathcal{S}_{H}^{0}$ be the subclass of $\mathcal{S}_{H}$ whose members $f$ satisfy the additional condition $f_{\bar{z}}(0)=0$. Let $\mathscr{K}_{H}^{0}$ and $\mathscr{C}_{H}^{0}$ be the subclasses of $\mathcal{S}_{H}^{0}$ whose image domains are convex and close to convex, respectively.

A domain $\Omega \in \mathbb{C}$ is said to be convex in the direction $\gamma, 0 \leq \gamma<\pi$, if every line parallel to the line joining 0 and $e^{i \gamma}$ has a connected intersection with $\Omega$. In particular, a domain is said to be convex in horizontal direction (CHD) if its intersection with each horizontal line is connected (or empty). In this paper, a function $f=h+\bar{g} \in \mathcal{S}_{H}^{0}$ is called a CHD mapping if $f$ maps $\mathbb{D}$ onto a CHD domain. We denote by $\mathcal{S}_{\mathrm{CHD}}^{0}$ the class of all CHD mappings. Clearly, $\mathcal{\delta}_{\mathrm{CHD}}^{0} \subset \mathscr{C}_{H}^{0}$.

The shear construction is essential to the present work as it allows one to study harmonic functions through their related analytic functions (see [3]); the shear construction produces a univalent harmonic function that maps $\mathbb{D}$ to a region, that is, CHD. This construction relies on the following theorem due to Clunie and Sheil-Small.

Theorem A (see [1]). A harmonic function $f=h+\bar{g}$ locally univalent in $\mathbb{D}$ is a univalent mapping of $\mathbb{D}$ onto a domain convex in the horizontal direction if and only if $h-g$ is a conformal univalent mapping of $\mathbb{D}$ onto a domain convex in the horizontal direction. 
For two harmonic functions; $f(z)=h(z)+\overline{g(z)}=$ $z+\sum_{n=2}^{\infty} a_{n} z^{n}+\sum_{n=1}^{\infty} \overline{b_{n}} \bar{z}^{n}$ and $F(z)=H(z)+\overline{G(z)}=z+$ $\sum_{n=2}^{\infty} A_{n} z^{n}+\sum_{n=1}^{\infty} \overline{B_{n}} \bar{z}^{n}$, their convolution is denoted by $f * F$ and defined as follows:

$$
\begin{aligned}
f * F & =h * H+\overline{g * G} \\
& =z+\sum_{n=2}^{\infty} a_{n} A_{n} z^{n}+\sum_{n=2}^{\infty} \overline{b_{n} B_{n}} \bar{z}^{n} .
\end{aligned}
$$

One can find the recent results involving harmonic convolutions in $[4-8]$. In $[9,10]$, explicit descriptions are given for half-plane and strip mappings. Specifically, the collection of the mappings $f=h+\bar{g} \in \mathcal{S}_{H}^{0}$ that map $\mathbb{D}$ onto the right half-plane, $\mathbb{M}=\{\omega: \operatorname{Re}(\omega)>-1 / 2\}$. Such mappings satisfy the condition

$$
h(z)+g(z)=\frac{z}{1-z} .
$$

In [4], the following result was derived.

Theorem B. Let $f_{1}=h_{1}+\overline{g_{1}}, f_{2}=h_{2}+\overline{g_{2}} \in \mathscr{K}_{H}^{0}$ be the right half-plane mappings. If $f_{1} * f_{2}$ is locally univalent and sense-preserving, then $f_{1} * f_{2} \in \mathcal{S}_{C H D}^{0}$.

Let $f_{0}=h_{0}+\overline{g_{0}}$ be the canonical right half-plane mapping with the dilatation $\omega_{0}=g_{0}^{\prime} / h_{0}^{\prime}=-z$; then

$$
h_{0}=\frac{z-(1 / 2) z^{2}}{(1-z)^{2}}, \quad g_{0}=\frac{-(1 / 2) z^{2}}{(1-z)^{2}} .
$$

Recently, Dorff et al. [5] obtained some results involving convolutions of $f_{0}$ with right half-plane mappings and vertical strip mappings. They proved the following.

Theorem C. Let $f=h+\bar{g} \in \mathscr{K}_{H}^{0}$ with $h+g=z /(1-z)$ and $\omega=g^{\prime} / h^{\prime}=e^{i \theta} z^{n}\left(n \in \mathbb{Z}^{+} ; \theta \in \mathbb{R}\right)$. If $n=1,2$, then $f_{0} * f \in \mathcal{S}_{C H D}^{0}$.

Theorem D. Let $f=h+\bar{g} \in \mathscr{K}_{H}^{0}$ with $h+g=$ $(1 / 2 i \sin \alpha) \log \left(\left(1+z e^{i \alpha}\right) /\left(1+z e^{-i \alpha}\right)\right)$, where $\pi / 2 \leq \alpha<\pi$ and $\omega=e^{i \theta} z^{n}$. If $n=1,2$, then $f_{0} * f \in \mathcal{S}_{C H D}^{0}$.

In this paper, we consider the harmonic mapping $P=h_{p}+$ $\overline{g_{p}}$ satisfies the condition

$$
h_{p}-g_{p}=\frac{z}{1-z}
$$

and when $\omega=z$, applying the shear construction, we have

$$
\begin{gathered}
h_{p}=\frac{z-(1 / 2) z^{2}}{(1-z)^{2}}=\frac{1}{2}\left(\frac{z}{(1-z)^{2}}+\frac{z}{1-z}\right), \\
g_{p}=\frac{(1 / 2) z^{2}}{(1-z)^{2}}=\frac{1}{2}\left(\frac{z}{(1-z)^{2}}-\frac{z}{1-z}\right) .
\end{gathered}
$$

Hence

$$
P(z)=\frac{1}{2}\left(\frac{z}{(1-z)^{2}}+\frac{z}{1-z}\right)+\frac{1}{2} \overline{\left(\frac{z}{(1-z)^{2}}-\frac{z}{1-z}\right)} .
$$

If $F$ is analytic in $\mathbb{D}$ and $F(0)=0$, then

$$
\begin{aligned}
& h_{p}(z) * F(z)=\frac{1}{2}\left[z F^{\prime}(z)+F(z)\right], \\
& g_{p}(z) * F(z)=\frac{1}{2}\left[z F^{\prime}(z)-F(z)\right] .
\end{aligned}
$$

We have known that $P(\mathbb{D})=\left\{u+i v: v^{2}>-(u+(1 / 4))\right\}$. The image of $\mathbb{D}$ under the harmonic mapping $P(z)$ is shown in Figure 1.

Writing $I(z)=z /(1-z)$, we can express $P(z)$ as

$$
P(z)=\frac{z I^{\prime}(z)+I(z)}{2}+\overline{\left(\frac{z I^{\prime}(z)-I(z)}{2}\right)} .
$$

The generalization is done in the following way. For $f$ : $\mathbb{D} \rightarrow \mathbb{C}$ a univalent analytic function with $f(0)=f^{\prime}(0)-1=$ 0 , define

$$
\begin{aligned}
P_{c}[f](z)= & \frac{c z f^{\prime}(z)+f(z)}{1+c} \\
& +\overline{\left(\frac{c z f^{\prime}(z)-f(z)}{1+c}\right)}, \quad(z \in \mathbb{D}, c>0) .
\end{aligned}
$$

Clearly, $P_{1}[I]=P$.

Obviously,

$$
\begin{aligned}
P_{c}[I](z) & =H_{0}(z)+\overline{G_{0}(z)} \\
& =\frac{c z I^{\prime}(z)+I(z)}{1+c}+\overline{\left(\frac{c z I^{\prime}(z)-I(z)}{1+c}\right)} \\
& =\operatorname{Re}\left\{\frac{2 c z}{(1+c)(1-z)^{2}}\right\}+i \operatorname{Im}\left\{\frac{2 z}{(1+c)(1-z)}\right\} \\
& :=U+i V .
\end{aligned}
$$

Hence

$$
V^{2}=-\left(\frac{2 c}{1+c} U+\frac{1}{(1+c)^{2}}\right) \text {. }
$$

So $P_{c}[I](\mathbb{D})=\left\{U+i V: V^{2}>-\left((2 c /(1+c)) U+\left(1 /(1+c)^{2}\right)\right), c>\right.$ $0\}$.

If $F$ is analytic in $\mathbb{D}$ and $F(0)=0$, then we have

$$
\begin{aligned}
& H_{0}(z) * F(z)=\frac{1}{1+c}\left[c z F^{\prime}(z)+F(z)\right], \\
& G_{0}(z) * F(z)=\frac{1}{1+c}\left[c z F^{\prime}(z)-F(z)\right] .
\end{aligned}
$$

In [5], authors showed that Theorems C and D do not hold for $n \geq 3$. In the present paper, we construct a new subclass of harmonic mappings $P_{c}[f]$ defined by (11). In Section 2, we show that convolutions of $P_{c}[I]$ with $f_{n}=h+\bar{g}$ (where $h-g=z /(1-z)$ and dilatation $\omega(z)=e^{i \theta} z^{n}(\theta \in \mathbb{R}, n \in$ $\left.\mathbb{N}^{+}\right)$) are in the subclass $\delta_{\text {CHD }}^{0}$ for $0<c \leq 2 / n$ and for 


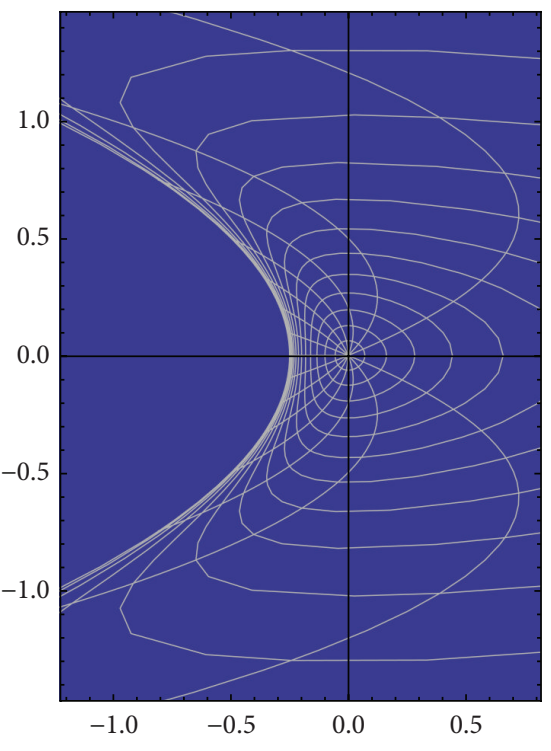

FIGURE 1: Image of $P(z)$.

all $n \in \mathbb{N}^{+}$. In Section 3, we apply the transformation to a normalized analytic univalent function $f$ and show that the sufficient and necessary condition for $P_{c}[f] \in \delta_{\mathrm{CHD}}^{0}$ is that $f$ is convex. Furthermore, we present some basic examples of harmonic mappings satisfying the conditions of the theorems and illustrate them graphically with the help of the Mathematica software. With these examples we explain the behavior of the image domains.

\section{The Convolution of $P_{c}[I]$}

To prove our main results, we need several lemmas.

Lemma 1 (see [11]). Let $f$ be an analytic function in $\mathbb{D}$ with $f(0)=0$ and $f^{\prime}(0) \neq 0$, and let

$$
\varphi(z)=\frac{z}{\left(1+z e^{i \theta_{1}}\right)\left(1+z e^{i \theta_{2}}\right)},
$$

where $\theta_{1}, \theta_{2} \in \mathbb{R}$. If

$$
\operatorname{Re}\left(\frac{z f^{\prime}(z)}{\varphi(z)}\right)>0 \quad(z \in \mathbb{D})
$$

then $f$ is convex in the horizontal direction.

Lemma 2. Let $P_{c}[I]$ be a mapping defined by (12) and $f=$ $h+\bar{g} \in \mathcal{S}_{H}$ with $h-g=z /(1-z)$ and dilatation $\omega(z)=$ $\left(g^{\prime}(z) / h^{\prime}(z)\right)\left(h^{\prime}(z) \neq 0, z \in \mathbb{D}\right)$. Then $\widetilde{\omega}_{1}$, the dilatation of $P_{c}[I] * f$, is given by

$$
\widetilde{\omega}_{1}=\frac{[(c-1)+(c+1) z] \omega(1-\omega)+c z \omega^{\prime}(1-z)}{[(c+1)+(c-1) z](1-\omega)+c z \omega^{\prime}(1-z)} .
$$

Proof. Since $h-g=z /(1-z)$ and $g^{\prime}=\omega h^{\prime}$, then $g^{\prime \prime}=$ $\omega^{\prime} h^{\prime}+\omega h^{\prime \prime}$. We immediately get

$$
\begin{gathered}
h^{\prime}=\frac{1}{(1-\omega)(1-z)^{2}}, \\
h^{\prime \prime}=\frac{2(1-\omega)+\omega^{\prime}(1-z)}{(1-\omega)^{2}(1-z)^{3}} .
\end{gathered}
$$

From (14), we have

$$
\begin{aligned}
\widetilde{\omega}_{1} & =\frac{\left(G_{0} * g\right)^{\prime}}{\left(H_{0} * h\right)^{\prime}}=\frac{\left(c z g^{\prime}-g\right)^{\prime}}{\left(c z h^{\prime}+h\right)^{\prime}}=\frac{(c-1) g^{\prime}+c z g^{\prime \prime}}{(c+1) h^{\prime}+c z h^{\prime \prime}} \\
& =\frac{(c-1) \omega h^{\prime}+c z\left(\omega^{\prime} h^{\prime}+\omega h^{\prime \prime}\right)}{(c+1) h^{\prime}+c z h^{\prime \prime}} \\
& =\frac{[(c-1)+(c+1) z] \omega(1-\omega)+c z \omega^{\prime}(1-z)}{[(c+1)+(c-1) z](1-\omega)+c z \omega^{\prime}(1-z)}
\end{aligned}
$$

Lemma 3. Let $f_{1}=h_{1}+\overline{g_{1}} \in \mathcal{S}_{H}^{0}$ with $h_{1}-g_{1}=z /(1-z)$. If $P_{c}[I] * f_{1}$ is locally univalent, then $P_{c}[I] * f_{1} \in \mathcal{S}_{C H D}^{0}$.

Proof. Recall that $P_{c}[I]=H_{0}+\overline{G_{0}}$ and

$$
H_{0}-G_{0}=\frac{2 z}{(1+c)(1-z)}, \quad h_{1}-g_{1}=\frac{z}{1-z} .
$$

Hence

$$
\begin{aligned}
h_{1}+g_{1} & =\frac{1+c}{2}\left(H_{0}-G_{0}\right) *\left(h_{1}+g_{1}\right) \\
& =\frac{1+c}{2}\left(H_{0} * h_{1}+H_{0} * g_{1}-G_{0} * h_{1}-G_{0} * g_{1}\right), \\
H_{0}+G_{0} & =\left(H_{0}+G_{0}\right) *\left(h_{1}-g_{1}\right) \\
& =\left(H_{0} * h_{1}-H_{0} * g_{1}+G_{0} * h_{1}-G_{0} * g_{1}\right) .
\end{aligned}
$$

Thus

$$
H_{0} * h_{1}-G_{0} * g_{1}=\frac{1}{2}\left[\frac{2}{1+c}\left(h_{1}+g_{1}\right)+\left(H_{0}+G_{0}\right)\right] \text {. }
$$

Next, we will show that $(2 /(1+c))\left(h_{1}+g_{1}\right)+\left(H_{0}+G_{0}\right)$ is convex in the horizontal direction.

Letting $\varphi(z)=z /(1-z)^{2} \in S^{*}$, we have

$$
\begin{aligned}
& \operatorname{Re}\left\{\frac{z\left[(2 /(1+c))\left(h_{1}^{\prime}+g_{1}^{\prime}\right)+\left(H_{0}^{\prime}+G_{0}^{\prime}\right)\right]}{\varphi}\right\} \\
& =\operatorname{Re}\left\{z\left[\frac{2}{1+c}\left(h_{1}^{\prime}-g_{1}^{\prime}\right)\left(\frac{h_{1}^{\prime}+g_{1}^{\prime}}{h_{1}^{\prime}-g_{1}^{\prime}}\right)+\left(H_{0}^{\prime}-G_{0}^{\prime}\right)\left(\frac{H_{0}^{\prime}+G_{0}^{\prime}}{H_{0}^{\prime}-G_{0}^{\prime}}\right)\right]\right. \\
& \left.\quad \times(\varphi)^{-1}\right\}
\end{aligned}
$$




$$
\begin{aligned}
&= \operatorname{Re}\left\{z\left[\frac{2}{1+c}\left(h_{1}^{\prime}-g_{1}^{\prime}\right)\left(\frac{1+\omega_{1}}{1-\omega_{1}}\right)+\left(H_{0}^{\prime}-G_{0}^{\prime}\right)\left(\frac{1+\omega_{0}}{1-\omega_{0}}\right)\right]\right. \\
&\left.\times(\varphi)^{-1}\right\} \\
&=\frac{2}{1+c} \operatorname{Re}\left\{\frac{\left(z /(1-z)^{2}\right)\left[p_{1}(z)+p_{0}(z)\right]}{z /(1-z)^{2}}\right\} \\
&=\frac{2}{1+c} \operatorname{Re}\left\{p_{1}(z)+p_{0}(z)\right\}>0,
\end{aligned}
$$

where $\omega_{1}=g_{1}^{\prime} / h_{1}^{\prime}, \omega_{0}=G_{0}^{\prime} / H_{0}^{\prime}$ and $p_{1}(z)=\left(1+\omega_{1}\right) /\left(1-\omega_{1}\right)$, $p_{0}(z)=\left(1+\omega_{0}\right) /\left(1-\omega_{0}\right)$. So $(2 /(1+c))\left(h_{1}+g_{1}\right)+\left(H_{0}+G_{0}\right)$ is convex in the horizontal direction by Lemma 1 , and then $H_{0} * h_{1}-G_{0} * g_{1}$ is convex in the horizontal direction. Finally, since we assumed that $P_{c}[I] * f_{1}$ is locally univalent, we apply Theorem A to get the desired result.

Now we turn to the distribution theory of the roots of polynomial for the unit disc. Given a polynomial of degree $n$ :

$$
\begin{aligned}
p(z) & =p_{0}(z) \\
& =a_{n, 0} z^{n}+a_{n-1,0} z^{n-1}+\cdots+a_{1,0} z+a_{0,0} \quad\left(a_{n, 0} \neq 0\right)
\end{aligned}
$$

with complex coefficients, the parallel algorithm for finding zeros of polynomial (24) inside the unit disc $\mathbb{D}$ is worth studying. Let

$$
\begin{aligned}
p^{*}(z) & =p_{0}^{*}(z) \\
& =z^{n} \overline{p\left(\frac{1}{\bar{z}}\right)} \\
& =\overline{a_{n, 0}}+\overline{a_{n-1,0}} z+\cdots+\overline{a_{1,0}} z^{n-1}+\overline{a_{0,0}} z^{n},
\end{aligned}
$$

it is easy to verify that the zeros of (24) and (25) are inverse points with respect to the unit disc $\mathbb{D}$. To derive the main judging theorem, we need the following lemma.

Lemma 4 (see [12]). If all zeros of (24) are inside the unit disc $\mathbb{D}$, then $\left|a_{0,0}\right|<\left|a_{n, 0}\right|$.

By Lemma 4, we can obtain the following result.

Lemma 5. If $\left|a_{0,0}\right| \geq\left|a_{n, 0}\right|$, then not all zeros of (24) are inside the unit disc $\mathbb{D}$.

Next, we construct a function sequence

$$
\left\{p_{k}(z) \mid k=0,1, \ldots, m,(m \leq n)\right\},
$$

where

$$
\begin{aligned}
& p_{k}(z)=a_{n-k, k} z^{n-k}+a_{n-k-1, k} z^{n-k-1}+\cdots+a_{1, k} z+a_{0, k}, \\
& p_{k}^{*}(z)=\overline{a_{n-k, k}}+\overline{a_{n-k-1, k}} z+\cdots+\overline{a_{1, k}} z^{n-k-1}+\overline{a_{0, k}} z^{n-k},
\end{aligned}
$$

$$
p_{k+1}(z)= \begin{cases}\frac{p_{k}(z)}{z}, & a_{n-k, k}=0, \\ \frac{a_{n-k, k} p_{k}(z)-a_{0, k} p_{k}^{*}(z)}{z}, & a_{n-k, k} \neq 0,\end{cases}
$$

where $k=0,1,2, \ldots, m-1$. If $\left|a_{0, k}\right| \geq\left|a_{n-k, k}\right|$, then by Lemma 5 we know that not all roots of $p_{k}(z)=0$ are inside the unit disc $\mathbb{D}$; we would not need to construct the function $p_{k+1}(z)$. If $\left|a_{0, k}\right|<\left|a_{n-k, k}\right|$, then use (28) to construct (26). The following lemma is a necessary and sufficient condition of all zeros of (24) lying inside the unit disc.

Lemma 6 (see [12]). A necessary and sufficient condition that all zeros of (24) inside the unit disc $\mathbb{D}$ is $\left|a_{0, k}\right|<\left|a_{n-k, k}\right|$ ( $k=$ $0,1, \ldots, n-1)$, where $a_{0, k}$ and $a_{n-k, k}$ are given by (27).

The main result of this section is the following.

Theorem 7. Let $P_{c}[I]$ be a mapping defined by (12) and $f_{n}=$ $h+\bar{g}$ with $h-g=z /(1-z)$ and dilatation $\omega(z)=e^{i \theta} z^{n}(\theta \in$ $\mathbb{R}, n \in \mathbb{N})$. Then $P_{c}[I] * f_{n} \in \mathcal{S}_{C H D}^{0}$ for $0<c \leq 2 / n$.

Proof. In view of Lemma 3, it suffices to show that the dilatation of $P_{c}[I] * f_{n}$ satisfies $\left|\widetilde{\omega}_{1}(z)\right|<1$, for all $z \in \mathbb{D}$. Setting $\omega(z)=e^{i \theta} z^{n}$ into (17), we get

$$
\begin{aligned}
\widetilde{\omega}_{1}= & \left([(c-1)+(c+1) z] e^{i \theta} z^{n}\left(1-e^{i \theta} z^{n}\right)+c n e^{i \theta} z^{n}(1-z)\right) \\
& \times\left([(c+1)+(c-1) z]\left(1-e^{i \theta} z^{n}\right)+c n e^{i \theta} z^{n}(1-z)\right)^{-1} \\
= & -e^{2 i \theta} z^{n} \\
& \times\left(\left(z^{n+1}-\frac{1-c}{1+c} z^{n}-\frac{1-(n-1) c}{1+c} e^{-i \theta} z+\frac{1-(n+1) c}{1+c} e^{-i \theta}\right)\right. \\
& \times\left(1-\frac{1-c}{1+c} z-\frac{1-(n-1) c}{1+c} e^{i \theta} z^{n}\right. \\
& \left.\left.\quad+\frac{1-(n+1) c}{1+c} e^{i \theta} z^{n+1}\right)^{-1}\right) \\
= & -e^{2 i \theta} z^{n} \frac{p(z)}{p^{*}(z)},
\end{aligned}
$$

where

$$
\begin{aligned}
p(z)= & z^{n+1}-\frac{1-c}{1+c} z^{n} \\
& -\frac{1-(n-1) c}{1+c} e^{-i \theta} z+\frac{1-(n+1) c}{1+c} e^{-i \theta}
\end{aligned}
$$

and $p^{*}(z)=z^{n+1} \overline{p(1 / \bar{z})}$.

Obviously, if $z_{0}$ is a zero of $p(z)$, the $1 / \overline{z_{0}}$ is a zero of $p^{*}(z)$. Hence, if $A_{1}, A_{2}, \ldots, A_{n+1}$ are the zeros of $p(z)$, we can write

$$
\widetilde{\omega}_{1}=-e^{2 i \theta} z^{n} \frac{\left(z-A_{1}\right)}{\left(1-\overline{A_{1}} z\right)} \cdot \frac{\left(z-A_{2}\right)}{\left(1-\overline{A_{2}} z\right)} \cdots \frac{\left(z-A_{n+1}\right)}{\left(1-\overline{A_{n+1}} z\right)} .
$$

Now for $\left|A_{i}\right| \leq 1,\left(\left(z-A_{i}\right) /\left(1-\overline{A_{i}} z\right)\right)(i=1,2, \ldots, n+1)$ maps $\overline{\mathbb{D}}$ onto $\overline{\mathbb{D}}$. So in order to prove our result, we will show that all zeros of $p(z)$ named $A_{1}, A_{2}, \ldots, A_{n+1}$ lie inside the 
unit disc $\overline{\mathbb{D}}$ for $0<c \leq 2 / n$. In the following we divide our proof in two cases.

Case 1 (when $c=2 / n)$. In this case, substituting $c=2 / n$ into (29), we see that

$$
\begin{aligned}
\left|\widetilde{\omega}_{1}\right|=\mid-e^{2 i \theta} z^{n}( & \left(z^{n+1}-\frac{n-2}{n+2} z^{n}+\frac{n-2}{n+2} e^{-i \theta} z-e^{-i \theta}\right) \\
& \left.\times\left(1-\frac{n-2}{n+2} z+\frac{n-2}{n+2} e^{i \theta} z^{n}-e^{i \theta} z^{n+1}\right)^{-1}\right) \mid \\
=\mid e^{i \theta} z^{n}( & \left(-e^{i \theta} z^{n+1}+\frac{n-2}{n+2} e^{i \theta} z^{n}-\frac{n-2}{n+2} z+1\right) \\
& \left.\times\left(1-\frac{n-2}{n+2} z+\frac{n-2}{n+2} e^{i \theta} z^{n}-e^{i \theta} z^{n+1}\right)^{-1}\right) \mid \\
= & \left|e^{i \theta} z^{n}\right|<1 .
\end{aligned}
$$

Case 2 (when $0<c<2 / n$ ). From (31), it is enough to show that all zeros of (30) are inside the unit disc $\overline{\mathbb{D}}$ for $0<c<2 / n$. Since $\left|a_{0,0}\right|=\left|((1-(n+1) c) /(1+c)) e^{-i \theta}\right|<1=\left|a_{n+1,0}\right|$, we can apply (28) to $p(z)$; thus we have

$$
\begin{aligned}
p_{1}(z)= & \frac{\overline{a_{n+1,0}} p(z)-a_{0,0} p^{*}(z)}{z} \\
= & \frac{p(z)-((1-(n+1) c) /(1+c)) e^{-i \theta} p^{*}(z)}{z} \\
= & \frac{(n+2) c(2-n c)}{(1+c)^{2}} z^{n}-\frac{n c(2-n c)}{(1+c)^{2}} z^{n-1} \\
& -\frac{2 c(2-n c)}{(1+c)^{2}} e^{-i \theta} \\
= & \frac{(n+2) c(2-n c)}{(1+c)^{2}}\left(z^{n}-\frac{n}{n+2} z^{n-1}-\frac{2}{n+2} e^{-i \theta}\right) \\
= & \frac{(n+2) c(2-n c)}{(1+c)^{2}} q_{1}(z),
\end{aligned}
$$

where $q_{1}(z)=z^{n}-(n /(n+2)) z^{n-1}-(2 /(n+2)) e^{-i \theta}$ and $q_{1}^{*}(z)=$ $1-(n /(n+2)) z-(2 /(n+2)) e^{i \theta} z^{n}$.

Since $\left|a_{0,1}\right|=\left|-(2 /(n+2)) e^{-i \theta}\right|=2 /(n+2)<1=\left|a_{n, 1}\right|$ for $n \geq 1$, by using (28) on $q_{1}(z)$ again, we get

$$
\begin{aligned}
p_{2}(z) & =\frac{\overline{a_{n, 1}} q_{1}(z)-a_{0,1} q_{1}^{*}(z)}{z} \\
& =\frac{q_{1}(z)+(2 /(n+2)) e^{-i \theta} q_{1}^{*}(z)}{z} \\
& =\frac{n(n+4)}{(n+2)^{2}}\left(z^{n-1}-\frac{n+2}{n+4} z^{n-2}-\frac{2}{n+4} e^{-i \theta}\right) \\
& =\frac{n(n+4)}{(n+2)^{2}} q_{2}(z),
\end{aligned}
$$

where $q_{2}(z)=z^{n-1}-((n+2) /(n+4)) z^{n-2}-(2 /(n+4)) e^{-i \theta}$ and $q_{2}^{*}(z)=1-((n+2) /(n+4)) z-(2 /(n+4)) e^{i \theta} z^{n-1}$.
Since $\left|a_{0,2}\right|=\left|-(2 /(n+4)) e^{i \theta}\right|=2 /(n+4)<1=\left|a_{n-1,2}\right| ;$ we have

$$
\begin{aligned}
p_{3}(z) & =\frac{\overline{a_{n-1,2}} q_{2}(z)-a_{0,2} q_{2}^{*}(z)}{z} \\
& =\frac{q_{1}(z)+(2 /(n+4)) e^{-i \theta} q_{1}^{*}(z)}{z} \\
& =\frac{(n+2)(n+6)}{(n+4)^{2}}\left(z^{n-2}-\frac{n+4}{n+6} z^{n-3}-\frac{2}{n+6} e^{-i \theta}\right) \\
& =\frac{(n+2)(n+6)}{(n+4)^{2}} q_{3}(z) .
\end{aligned}
$$

Continuing in this manner we derive that

$$
\begin{aligned}
p_{k}(z)= & \frac{\overline{a_{n+1-k, k}} q_{k}(z)-a_{0, k} q_{k}^{*}(z)}{z} \\
= & \frac{[n+2(k-2)](n+2 k)}{[n+2(k-1)]^{2}} \\
& \times\left(z^{n+1-k}-\frac{n+2(k-1)}{n+2 k} z^{n-k}-\frac{2}{n+2 k} e^{-i \theta}\right) \\
= & \frac{[n+2(k-2)](n+2 k)}{[n+2(k-1)]^{2}} q_{k}(z) \quad(k=2,3, \ldots, n),
\end{aligned}
$$

where $q_{k}(z)=z^{n+1-k}-((n+2(k-1)) /(n+2 k)) z^{n-k}-(2 /(n+$ $2 k)) e^{-i \theta}$ and then $\left|a_{0, k}\right|=\left|-(2 /(n+2 k)) e^{-i \theta}\right|=2 /(n+2 k)<$ $1=\left|a_{n+1-k, k}\right|$. Hence, let $k=n$, we have

$$
p_{n}(z)=\frac{3 n(3 n-4)}{(3 n-2)^{2}}\left(z-\frac{3 n-2}{3 n}-\frac{2}{3 n} e^{-i \theta}\right) .
$$

If $\theta \neq 2 m \pi, m \in \mathbb{N}$, then $\left|a_{0, n}\right|=\left|\left(3 n-2+2 e^{-i \theta}\right) / 3 n\right|<1=$ $\left|a_{1, n}\right|$; by Lemma 6 , thus, $A_{1}, A_{2}, \ldots, A_{n+1}$ lie inside unit disc.

If $\theta=2 m \pi, m \in \mathbb{N}$, then $p(z)=z^{n+1}-((1-c) /(1+c)) z^{n}-$ $((1-(n-1) c) /(1+c)) z+((1-(n+1) c) /(1+c))$. As $z=1$ is a zero of $p(z)$, therefore we can write

$$
\begin{aligned}
p(z)= & (z-1) \\
\times & {\left[z^{n}+\left(1-\frac{1-c}{1+c}\right) z^{n-1}+\left(1-\frac{1-c}{1+c}\right) z^{n-2}\right.} \\
& \left.+\cdots+\left(1-\frac{1-c}{1+c}\right) z-\frac{1-(n+1) c}{1+c}\right] \\
= & (z-1) Q(z) .
\end{aligned}
$$

It suffices to show that zeros of $Q(z)$ lie inside unit disc. Since $|-(1-(n+1) c) /(1+c)|<1$ whenever $0<c<2 / n$, by applying (28) on $Q(z)$, we obtain analogously that all zeros of $Q(z)$ lie inside unit disc.

To sum up, we showed that all zeros of $p(z)$ lie inside or on the unit circle $|z|=1$ for $0<c \leq 2 / n$. The proof of our theorem is now completed. 


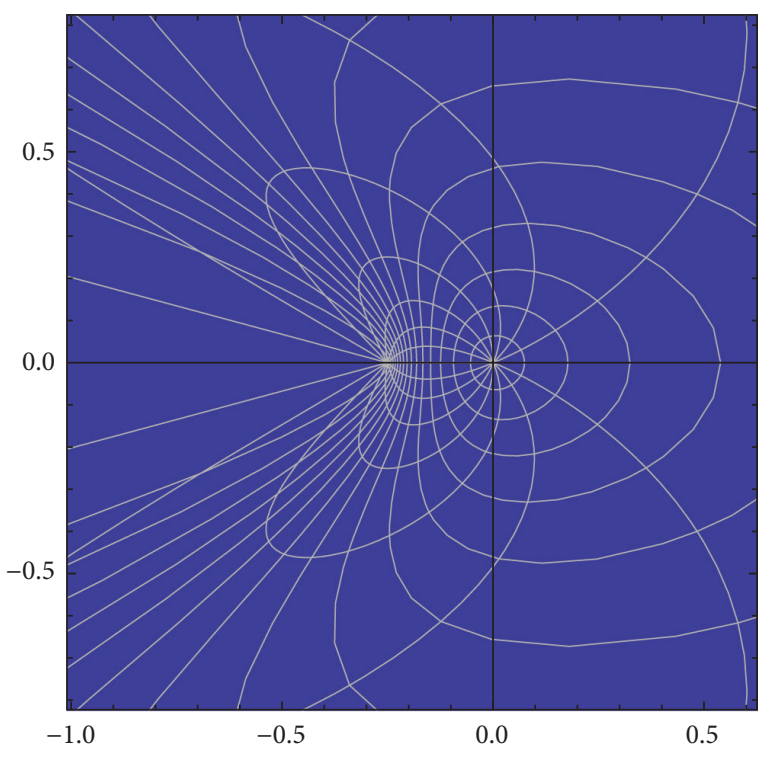

FIgURE 2: Image of $P(z) * P(z)$.

Next we give an example showing given values of $\theta, n$, and $c$ in Theorem 7.

Example 8. In Theorem 7, if we take $\theta=0, n=1$, then $h_{1}-$ $g_{1}=z /(1-z)$ and $\omega=z$, and we get $f_{1}=h_{1}+\overline{g_{1}}=P(z)=$ $\left(\left(z-(1 / 2) z^{2}\right) /(1-z)^{2}\right)+\left(\overline{(1 / 2) z^{2} /(1-z)^{2}}\right)$. Let $c=1$; then $P_{1}[I]=P(z)$ and $P_{1}[I] * f_{1}=P(z) * P(z)=H_{1}+\overline{G_{1}}$. From (7) and (9), we have

$$
\begin{gathered}
H_{1}=h_{p} * h_{p}=\frac{1}{2}\left[z h_{p}^{\prime}+h_{p}\right]=\frac{4 z-3 z^{2}+z^{3}}{4(1-z)^{3}}, \\
G_{1}=g_{p} * g_{p}=\frac{1}{2}\left[z g_{p}^{\prime}-g_{p}\right]=\frac{z^{2}+z^{3}}{4(1-z)^{3}} .
\end{gathered}
$$

The images of concentric circles inside $\mathbb{D}$ under the harmonic mapping $P(z)$ and concentric circles under the convolution map $P(z) * P(z)$ are shown in Figures 1 and 2, respectively.

\section{Harmonic Univalent Mappings Convex in One Direction}

For the local univalence of $P_{c}[f]$ defined by (11), we have the following.

Lemma 9. The function $P_{c}[f]$ defined by (11) is locally univalent if and only if $f$ is convex.

Proof. Write $P_{c}[f]=F=H+\bar{G}$. By (1), $F$ is locally univalent if and only if $|\omega(z)|=\left|G^{\prime}(z) / H^{\prime}(z)\right|<1$ for all $z \in \mathbb{D}$, and $F$ will be locally univalent if and only if

$$
|\omega(z)|=\left|\frac{(c-1) f^{\prime}(z)+c z f^{\prime \prime}(z)}{(c+1) f^{\prime}(z)+c z f^{\prime \prime}(z)}\right|<1,
$$

or equivalently, since $c>0$ and $f \in \mathcal{S}$,

$$
\left|\left(1+\frac{z f^{\prime \prime}(z)}{f^{\prime}(z)}\right)-\frac{1}{c}\right|<\left|\left(1+\frac{z f^{\prime \prime}(z)}{f^{\prime}(z)}\right)+\frac{1}{c}\right| .
$$

It can be easily seen that the inequality above is equivalent to

$$
\operatorname{Re}\left(1+\frac{z f^{\prime \prime}(z)}{f^{\prime}(z)}\right)>0
$$

which is the analytic condition for convexity. Thus, $P_{c}[f]$ is locally univalent if and only if $f$ is convex.

We immediately have the following result.

Theorem 10. The function $P_{c}[f] \in \mathcal{S}_{C H D}^{0}$ is defined by (11) if and only if $f$ is convex.

Proof. Again write $P_{c}[f]=F=H+\bar{G}$. Since $H-G=2 f /(1+$ c) is convex, particularly, $H-G$ is convex in the horizontal direction; by Lemma 9 and Theorem A, the proof is complete.

The following example satisfies the condition of Theorem 10.

Example 11. Consider the univalent analytic function $f(z)=$ $z+\left(z^{2} / 4\right)$; obviously, $f(0)=f^{\prime}(0)-1=0$, and

$$
\operatorname{Re}\left(1+\frac{z f^{\prime \prime}(z)}{f^{\prime}(z)}\right)=\operatorname{Re}\left(1+\frac{z / 2}{1+(z / 2)}\right)>0, \quad \text { for } z \in \mathbb{D} \text {. }
$$

So, $f(z)$ is convex analytic in $\mathbb{D}$. Therefore, in view of Theorem 10, the function $P_{c}[f](z) \in S_{\mathrm{CHD}}^{0}$. However

$$
P_{c}[f](z)=\operatorname{Re}\left(\frac{2 c z f^{\prime}(z)}{1+c}\right)+i \operatorname{Im}\left(\frac{2 f(z)}{1+c}\right)
$$

Now, we let $c=1$; then

$$
P_{1}[f](z)=\operatorname{Re}\left(z+\frac{z^{2}}{2}\right)+i \operatorname{Im}\left(z+\frac{z^{2}}{4}\right) .
$$

The images of $\mathbb{D}$ under $f(z)$ and $P_{1}[f](z)$ are shown in Figures 3 and 4, respectively. Obviously, we see that the image of $\mathbb{D}$ under $P_{1}[f](z)$ is convex in the horizontal direction.

\section{Conflict of Interests}

The authors declare that they have no competing interests.

\section{Authors' Contribution}

The authors completed the paper together. They also read and approved the final paper. 


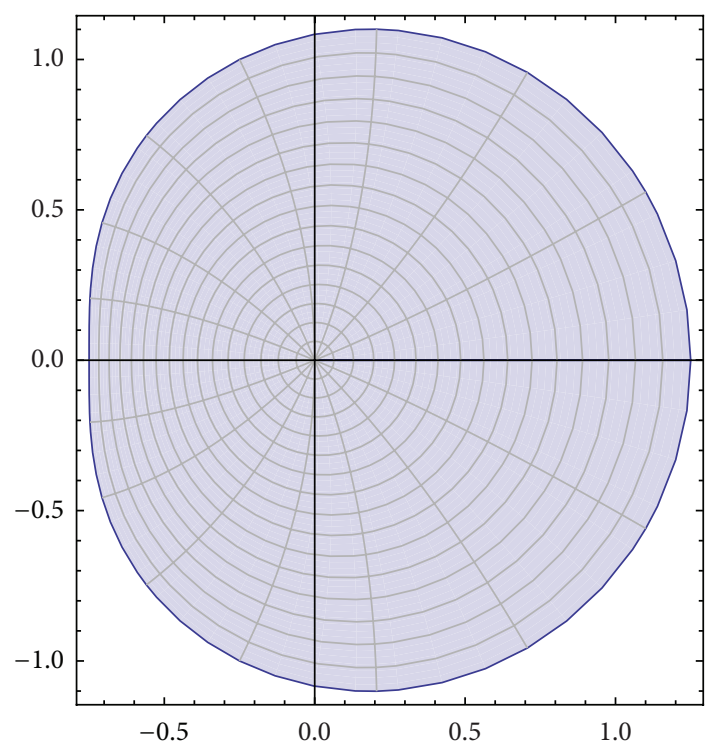

FIgURE 3: Image of $f(z)=z+z^{2} / 4$.

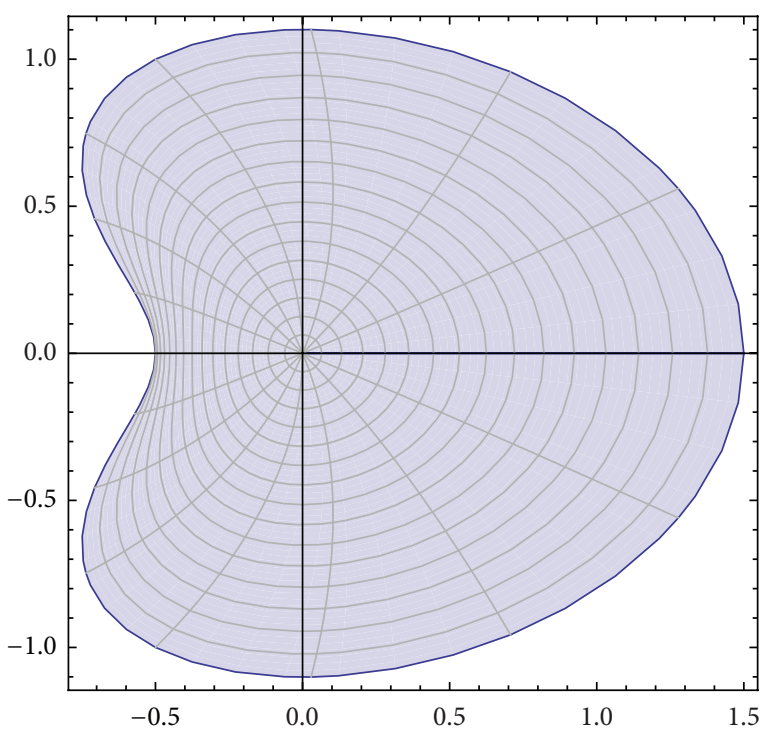

Figure 4: Image of $P_{1}[f](z)$.

\section{Acknowledgments}

The present investigation was supported by the National Natural Science Foundation under Grants 11301008 and 11226088 , the Key Courses Construction of Honghe University under Grant ZDKC1003 and the Scientific Research Fund of Yunnan Province under Grant 2010ZC150.

\section{References}

[1] J. Clunie and T. Sheil-Small, "Harmonic univalent functions," Annales Academiae Scientiarum Fennicae A, pp. 3-25, 1984.

[2] P. L. Duren, Univalent functions, vol. 259, Springer, New York, NY, USA, 1983.
[3] W. Hengartner and G. Schober, "On schlicht mappings to domains convex in one direction," Commentarii Mathematici Helvetici, vol. 45, pp. 303-314, 1970.

[4] M. Dorff, "Convolutions of planar harmonic convex mappings," Complex Variables, Theory and Application, vol. 45, no. 3, pp. 263-271, 2001.

[5] M. Dorff, M. Nowak, and M. Wołoszkiewicz, "Convolutions of harmonic convex mappings," Complex Variables and Elliptic Equations, vol. 57, no. 5, pp. 489-503, 2012.

[6] M. R. Goodloe, "Hadamard products of convex harmonic mappings," Complex Variables, Theory and Application, vol. 47, no. 2, pp. 81-92, 2002.

[7] L. L. Li and S. Ponnusamy, "Solution to an open problem on convolutions of harmonic mappings," Complex Variables and Elliptic Equations, 2012.

[8] L. L. Li and S. Ponnusamy, "Convolutions of slanted half-plane harmonic mappings," Analysis, vol. 33, pp. 159-176, 2013.

[9] Y. Abu-Muhanna and G. Schober, "Harmonic mappings onto convex domains," Canadian Journal of Mathematics, vol. 39, no. 6, pp. 1489-1530, 1987.

[10] W. Hengartner and G. Schober, "Univalent harmonic functions," Transactions of the American Mathematical Society, vol. 299, no. 1, pp. 1-31, 1987.

[11] C. Pommerenke, "On starlike and close-to-convex functions," Proceedings of the London Mathematical Society, vol. 13, pp. 290304, 1963.

[12] J. S. Cheng, "A parallel algorithm for finding roots of a complex polynomial," Journal of Computer Science and Technology, vol. 5, no. 1, pp. 71-81, 1990. 


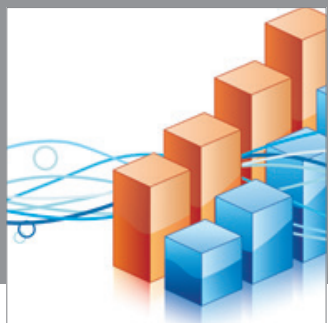

Advances in

Operations Research

mansans

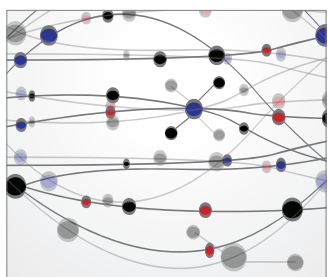

The Scientific World Journal
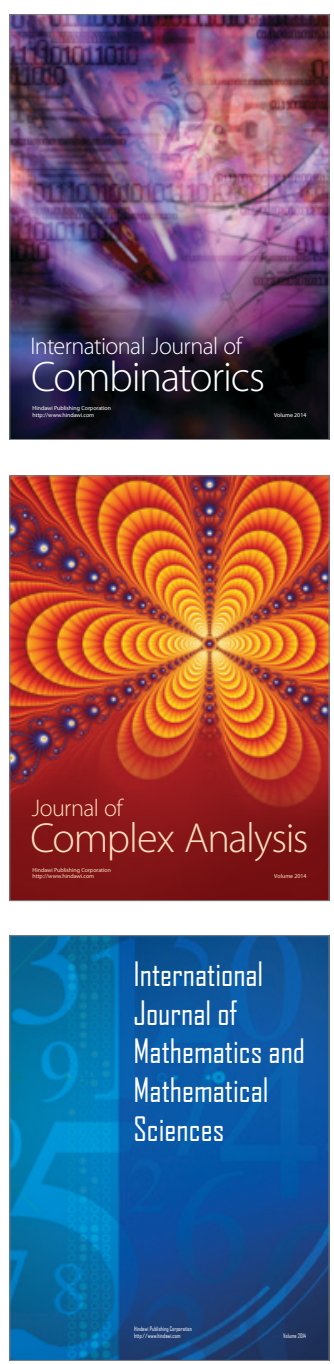
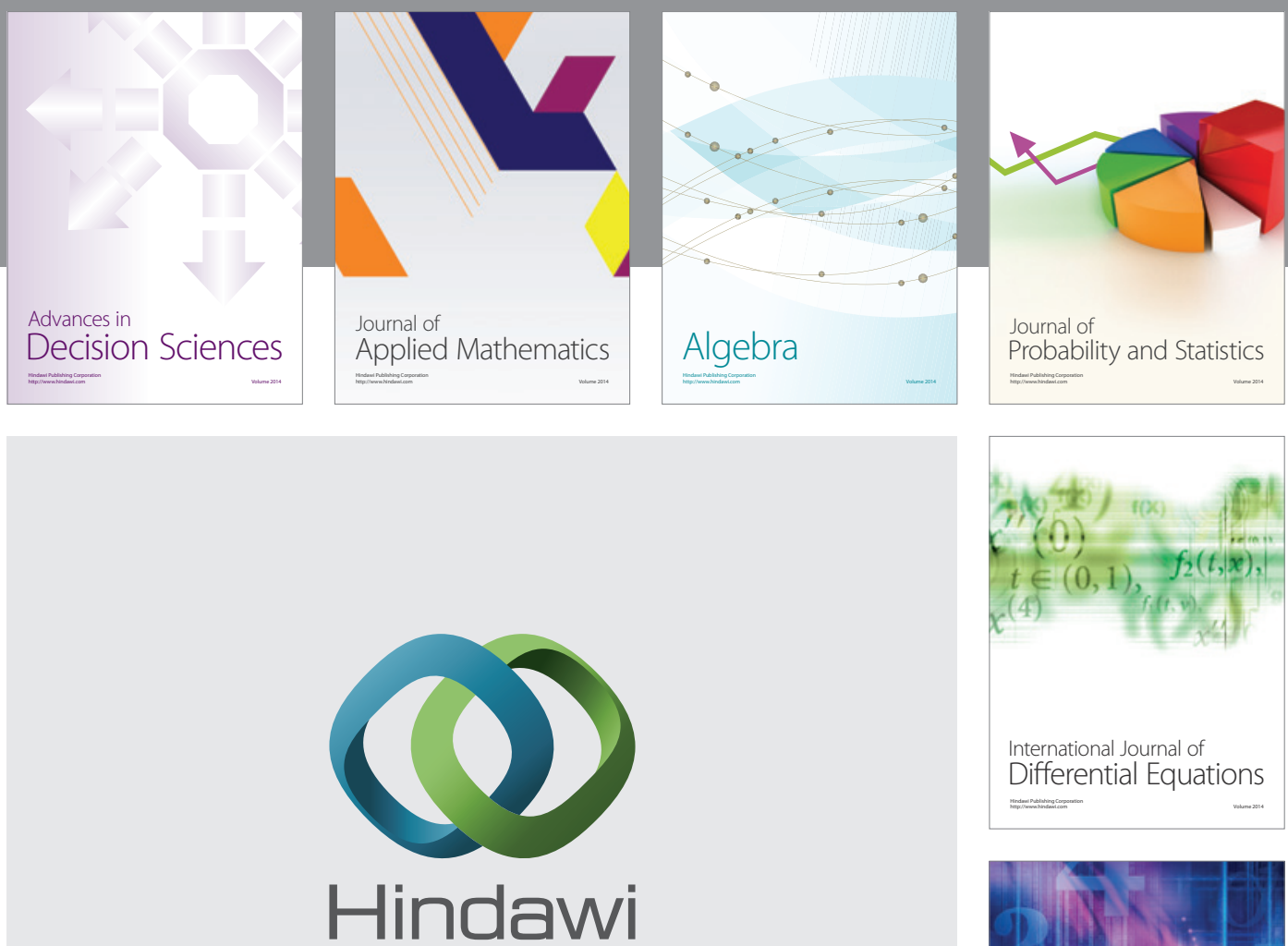

Submit your manuscripts at http://www.hindawi.com
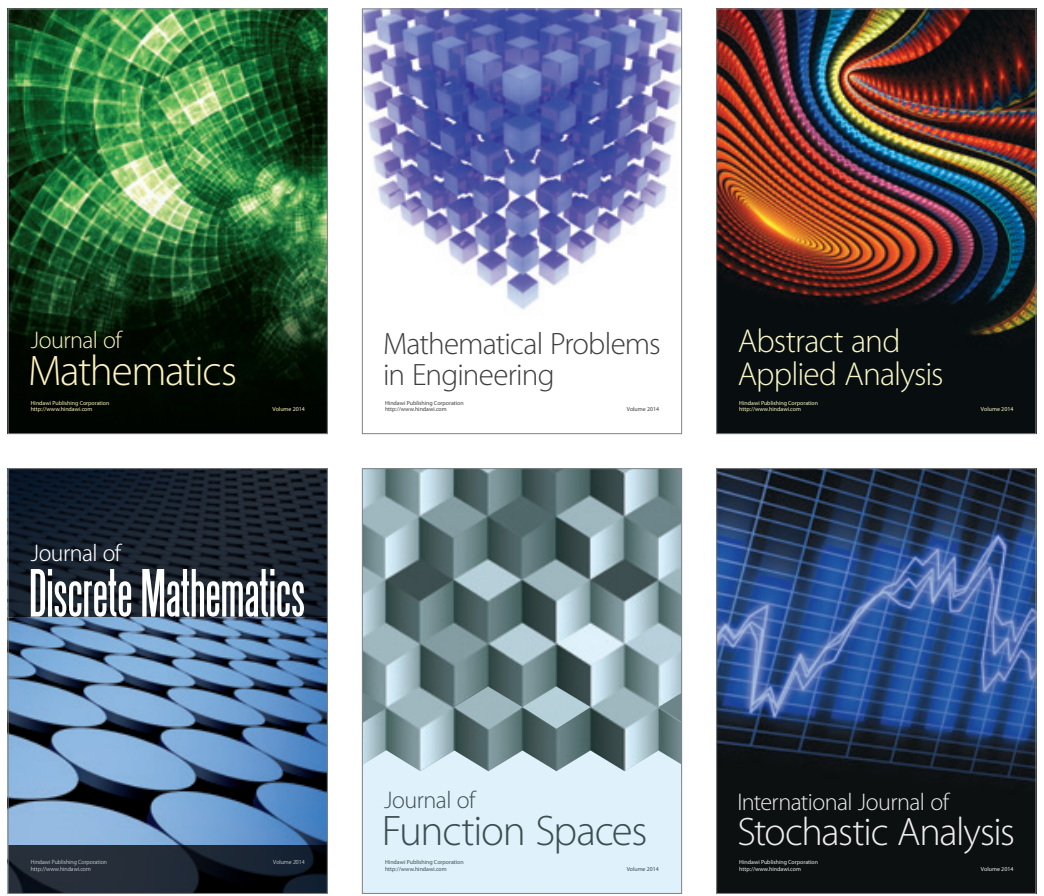

Journal of

Function Spaces

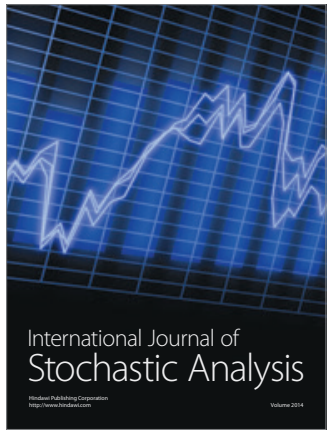

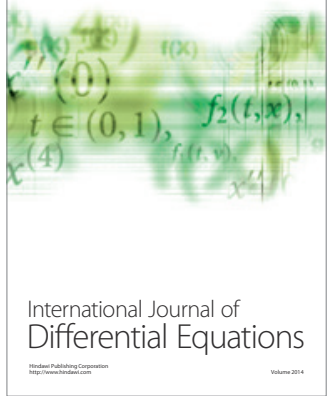
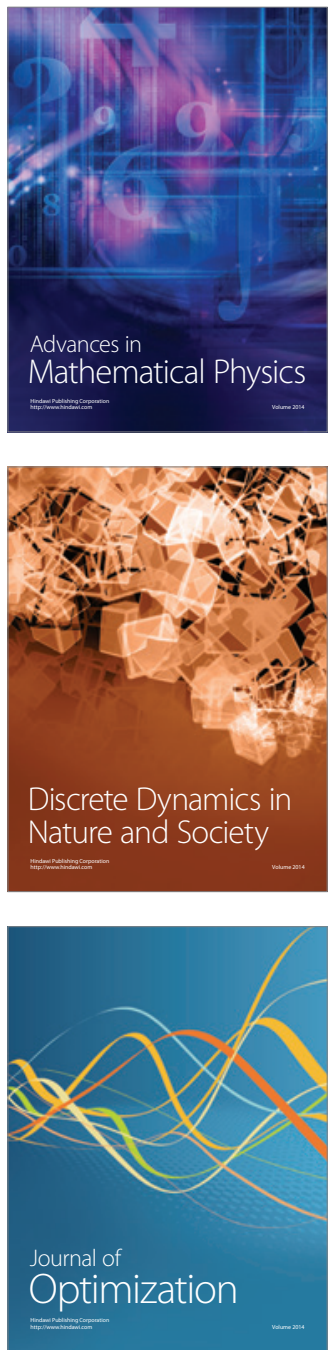